\title{
Recent progress at the Keck Interferometer
}

S. Ragland ${ }^{\mathrm{a},{ }^{*}}$, R. Akeson ${ }^{\mathrm{b}}$, M. Colavita ${ }^{\mathrm{c}}$, R. Millan-Gabet ${ }^{\mathrm{b}}$, T. Panteleeva ${ }^{\mathrm{a}}$, B. Smith ${ }^{\mathrm{a}}$, K. Summers ${ }^{\mathrm{a}}$, P. Wizinowich ${ }^{\mathrm{a}}$, J. Woillez $^{\mathrm{a}}$, E. Appleby ${ }^{\mathrm{a}}$, A. Cooper ${ }^{\mathrm{a}}$, C. Felizardo ${ }^{\mathrm{b}}$, J. Herstein ${ }^{\mathrm{b}}$, D. Morrison ${ }^{\mathrm{a}}$, K. Tsubota ${ }^{\mathrm{a}}$, C. Tyau ${ }^{\mathrm{a}}$

\author{
${ }^{a}$ W. M. Keck Observatory, 65-1120 Mamalahoa Hwy, Kamuela, HI 96743 \\ bASA Exoplanet Science Institute, California Institute of Technology, \\ 100-22, Pasadena, CA 91125 \\ ${ }^{\mathrm{c}}$ Jet Propulsion Laboratory, California Institute of Technology, \\ 4800 Oak Grove Dr., Pasadena, CA 91109
}

\begin{abstract}
The Keck Interferometer (KI) combines the two $10 \mathrm{~m}$ diameter Keck telescopes providing milliarcsecond angular resolution. $\mathrm{KI}$ has unique observing capabilities such as sensitive $\mathrm{K}$-band $\mathrm{V}^{2}$, $\mathrm{L}$-band $\mathrm{V}^{2}$ and N-band nulling modes. The instrument improvements and status of the Keck Interferometer since the 2010 SPIE meeting are summarized. We discuss the current capabilities of the KI, operational improvements, and the science from the KI during the past two years. We will conclude with a brief note on the closure of the KI facility. Details of dual field phase referencing developments and nulling science results are presented elsewhere at this conference.
\end{abstract}

Keywords: Interferometry, Visibility-squared, differtial phase, phase referencing, Nulling, L-band interferometry

\section{INTRODUCTION}

The Keck Interferometer (KI) has been operational since 2001, with shared risk science starting in June 2002, and formal commissioning of the first $\mathrm{V}^{2}$ modes in 2004. The progress of the KI is presented in several previous SPIE papers. ${ }^{1,2,3,4,5,6,7,8}$. The priorities of KI during the past two years have been the completion of the Dual-Field Phase Referencing (DFPR) mode of the ASTRA project and execution of TAC allocated science observations in various operational modes. In addition, several infrastructure and operational improvements have been made during this period in order to improve the observing efficiency and add new observing capabilities. In this paper, we discuss the progress at the Keck Interferometer in terms of operational capabilities, infrastructure and operational improvements, with special emphasis on what was implemented since the last SPIE meeting in San Diego, 2010.

The KI is a NASA funded project to perform long baseline interferometry with the two $10 \mathrm{~m}$ diameter Keck telescopes in the near and mid-infrared wavelengths. The physical baseline (B) of KI is $85 \mathrm{~m}$ providing an angular resolution $(\lambda / 2 \mathrm{~B})$ of $\sim 3$ mas at $2.2 \mu \mathrm{m} \& 12$ mas at $10 \mu \mathrm{m}$.

Since the 2010 SPIE meeting, KI operated on the sky for 50 nights - an average of $\sim 25$ nights per year. We continued to improve the observing efficiency of KI during this 2-year period through operations automation, preventive maintenance of the interferometer subsystems, staff training and updated procedures. KI is currently used for a variety of astrophysical studies, and there were 11 refereed publications during this period, including 10 papers on $\mathrm{V}^{2}$ observations and 2 papers on Nuller observations (one of the papers uses both $\mathrm{V}^{2}$ and Nuller observations).

In this paper, we describe the various operational capabilities of KI in Section 2, recent operational improvements in Section 3, highlights of science operations in Section 4, and a brief description of the plans for near future developments in Section 5.

Optical and Infrared Interferometry III, edited by Françoise Delplancke, Jayadev K. Rajagopal, Fabien Malbet, Proc. of SPIE Vol. 8445, 84450C · (c) 2012 SPIE · CCC code: 0277-786/12/\$18 - doi: 10.1117/12.925462 


\section{CURRENT CAPABILITIES}

The Keck Interferometer (KI) has unique operational capabilities such as sensitive $\mathrm{V}^{2}$ measurements in the $\mathrm{K}$ band, $\mathrm{V}^{2}$ measurements in the $\mathrm{L}_{\text {band }}{ }^{9}$, and nulling measurements in the $\mathrm{N}$ band ${ }^{10}$. Other operational capabilities include H-band $\mathrm{V}^{2}$ and high spectral resolution measurements through self-phase referencing $\left(\mathrm{V}^{2}-\mathrm{SPR}^{11}\right)$, simultaneous $\mathrm{V}^{2}$ measurements in the $\mathrm{K}$ and $\mathrm{L}$ bands $\left(\mathrm{V}^{2}-\mathrm{K} / \mathrm{L}\right.$ mode $\left.{ }^{9}\right)$ and $\mathrm{H}$ and $\mathrm{L}$ bands $\left(\mathrm{V}^{2}-\mathrm{H} / \mathrm{L}\right.$ mode $)$, and more recently, dual field phase referencing $\left(\mathrm{V}^{2}\right.$-DFPR $\left.{ }^{12}\right)$ capability. A complete list of all available operational modes of the KI is given in Table 1 along with the current performance.

\begin{tabular}{|l|l|l|}
\hline & KI Capabilities & Current performance \\
\hline 1 & Nulling mode & N-band flux $>1.7$ Jy \\
\hline 2 & $\mathrm{~V}^{2}-\mathrm{K} 5 ; \mathrm{R} \sim 27$ & $\mathrm{~K}^{\prime}<10.3$ \\
\hline 3 & $\mathrm{~V}^{2}-\mathrm{K} 10 ; \mathrm{R} \sim 54$ & $\mathrm{~K}^{\prime}<9.5$ \\
\hline 4 & $\mathrm{~V}^{2}-\mathrm{K} 42 ; \mathrm{R} \sim 230$ & $\mathrm{~K}^{\prime}<7.6$ \\
\hline 5 & $\mathrm{~V}^{2}-\mathrm{SPR} ; \mathrm{R} \sim 1700$ & $\mathrm{~K}^{\prime}<8$ \\
\hline 6 & $\mathrm{~V}^{2}-\mathrm{H} 4 ; \mathrm{R} \sim 22$ & $\mathrm{H}<9$ \\
\hline 7 & $\mathrm{~V}^{2}-\mathrm{L} 10 ; \mathrm{R} \sim 63$ & $\mathrm{~L}^{\prime}<6$ \\
\hline 8 & $\mathrm{~V}^{2}-\mathrm{K} / \mathrm{L}$ & $\mathrm{K}^{\prime}<8.7 \& \mathrm{~L}^{\prime}<4.8$ \\
\hline 9 & $\mathrm{~V}^{2}-\mathrm{H} / \mathrm{L}$ & $\mathrm{H}<8.0 \& \mathrm{~L}^{\prime}<4.8$ \\
\hline 10 & $\mathrm{~V}^{2}-$ DFPR & $\mathrm{K}<13$ for $3-25^{\prime \prime}$ field; guide star of $\mathrm{R}<14, \mathrm{H}<13 \& \mathrm{~K}<8$ \\
\hline
\end{tabular}

Table 1: Operational capabilities of the Keck Interferometer

The adaptive optics and angle tracking limits are as follows:

- AO sensitivity: $\mathrm{R}<14\left(\mathrm{R}<12\right.$ for Nuller, $\mathrm{V}^{2}-\mathrm{K} / \mathrm{L} \& \mathrm{~V}^{2}-\mathrm{H} / \mathrm{L}$ modes $)$

- KAT sensitivity: $\mathrm{J} / \mathrm{H}<10.5$ ( $\mathrm{H}<9$ for $\mathrm{SPR} \& \mathrm{H}<13$ for $\mathrm{V}^{2}$-DFPR $)$

\section{OPERATIONAL IMPROVEMENTS}

\subsection{New/Upgraded Operational Tools}

We have developed or improved several operational tools during the last two years. These new tools continue to improve our operational efficiency. Basic operational tools such as "IFoper" and "IFsys" underwent upgrades because of the addition of new subsystems, motors, picomotors, and shutters as a part of instrument development. Also, the sequencer software underwent significant changes. In the following sections, we briefly discuss a few significant improvements.

\subsubsection{Upgraded "IFoper" and "IFsys" Tools}

For the first several years of operation, the KI had just two modes $-\mathrm{V}^{2}$ and Nulling mode. It now supports several operational modes that are very different from each other. The new modes use different beam-combiners, switchyard optics, and science cameras. The IFoper GUI has been upgraded to allow us to switch between the shutters, light sources, filters, and the like, for beam-combiners $\mathrm{BC} 2$ and $\mathrm{BC}$. The devices monitored via IFoper have been extended to include devices used in the new configurations: the AO DFB corner cube position and off-axis field selector (OFS) targets and LEDs. 
The IFsys GUI was upgraded for the $\mathrm{V}^{2}$-SPR and $\mathrm{V}^{2}$-DFPR modes. Depending on the mode, the set of real-time CPUs used is different. Only four of the eleven real-time CPUs are used in every mode. Nulling mode is the only configuration that uses all eleven CPUs. The other modes use different combinations of fringe trackers and fast delay lines (and none of the three nuller CPUs). Adding the $\mathrm{V}^{2}$-SPR and $\mathrm{V}^{2}$-DFPR modes to the IFsys GUI allows us to see only the CPUs necessary for a given configuration. The GUI was also modified to start the sequencers for $\mathrm{V}^{2}$-SPR and $\mathrm{V}^{2}$-DFPR mode - previously these had to be started manually by the operator.

\subsubsection{Target Manager Tool}

A new Dual TargetManager GUI was developed enabling us to select targets for primary and secondary beam trains from the same GUI. Previously, separate target manager GUIs (and servers) were started - one for primary and one for secondary ${ }^{6}$.

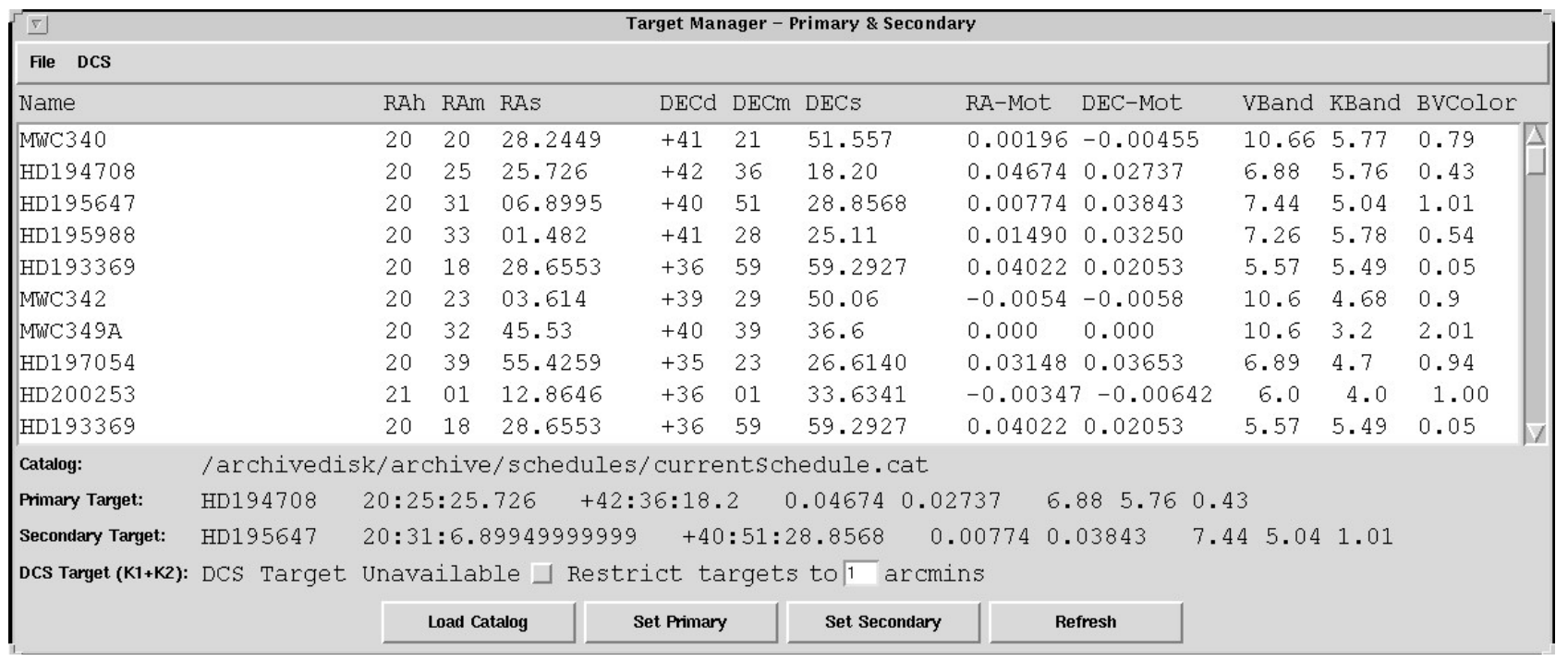

Figure 1: A screenshot of the TargetManager GUI

Additionally, both single and dual TargetManager GUIs were modified to get the telescope pointing information so that the operator may filter the list of targets shown based on proximity to the telescope pointing.

\subsubsection{Sequencer Upgrade}

The observing sequencers are the top-level software of the KI. The sequencers make the real-time components (fast delay lines, fringe trackers, etc.) and slow components (shutters, etc.) act in concert: sequencing through targets, performing calibrations, moving optics for acquisition, closing loops, etc.

Over the last two years, due to changes necessary for the ASTRA observing modes, the most significantly modified software has been the sequencers. The $\mathrm{V}^{2}$-DFPR Observing Sequencer, a new top-level sequencer, is now routinely used for $\mathrm{V}^{2}$-DFPR observations. In $\mathrm{V}^{2}$-DFPR mode the system is tracking two targets and the sequencer switches stars back and forth between two Fringe Trackers during sequences. The top-level sequencer keeps track of the target pair, and which target is brighter in K-band, so that it can swap correctly, change selected targets on the TargetManagers, set up the KAT configuration, fiber align on only the brighter star, and enable/disable the feed-forward between fringe trackers.

Updates to the low-level sequencers were necessary to allow the top-level Observing Sequencer to perform its required tasks. Care was taken to make sure the science observing modes were preserved when modifying sequencers for other modes. 
Changes made to the standard $\mathrm{V}^{2}$ sequencer include:

- Moved the raster states out of the standard integration sequence so that they can be performed from the toplevel sequencer on the brighter target on either beam combiner

- Made the standard set of calibrations optional

- Added timing of the integration sequence for system metrics

- Added new interfaces to allow the top-level sequencers to

- enable/disable feedforward on the fringe trackers, based on which sequencer is on the brighter target

$\circ$ track and idle the angle tracker

- maintain the angle tracker configuration (neutral density filters) when swapping targets

$\circ$ zero the FDL targets

Changes to other low-level sequencers, used in all observing modes (except Nulling) include:

- Split the FDL target generated into a beam train component and a sidereal component

- Added control of fringe tracker feedforward

- Added control of angle tracker neutral density filters

- Upgraded the TargetManager servers and FDL sequencers to use the Naval Observatory Vector Astrometry Software (NOVAS) library for calculating stellar positions and differences

- Bug fixes for catalog i/o.

The $\mathrm{V}^{2}$-DFPR mode Observing Sequencer is the most sophisticated sequencer implemented in the KI: it interfaces to an astrometric metrology sequencer, two instances of acquisition sequencers, and two instances of $\mathrm{V}^{2}$ sequencers. This is necessary because in $\mathrm{V}^{2}$-DFPR mode, there are two separate targets, a primary on-axis star and a secondary off-axis star, and the system needs to swap the two stars between the two interferometer beam trains during the sequence.

The acquisition sequencers control sequencers for the adaptive optics systems and the off-axis field selector (OFS) mirror systems. All three of these sequencers were newly written during the last two years. The $\mathrm{V}^{2}$ sequencers control the fringe tracker (FT), FDL, and angle tracker (KAT) sequencers. When the KI runs the $\mathrm{V}^{2}$-DFPR Observing Sequencer, there are 20 sequencer processes running!

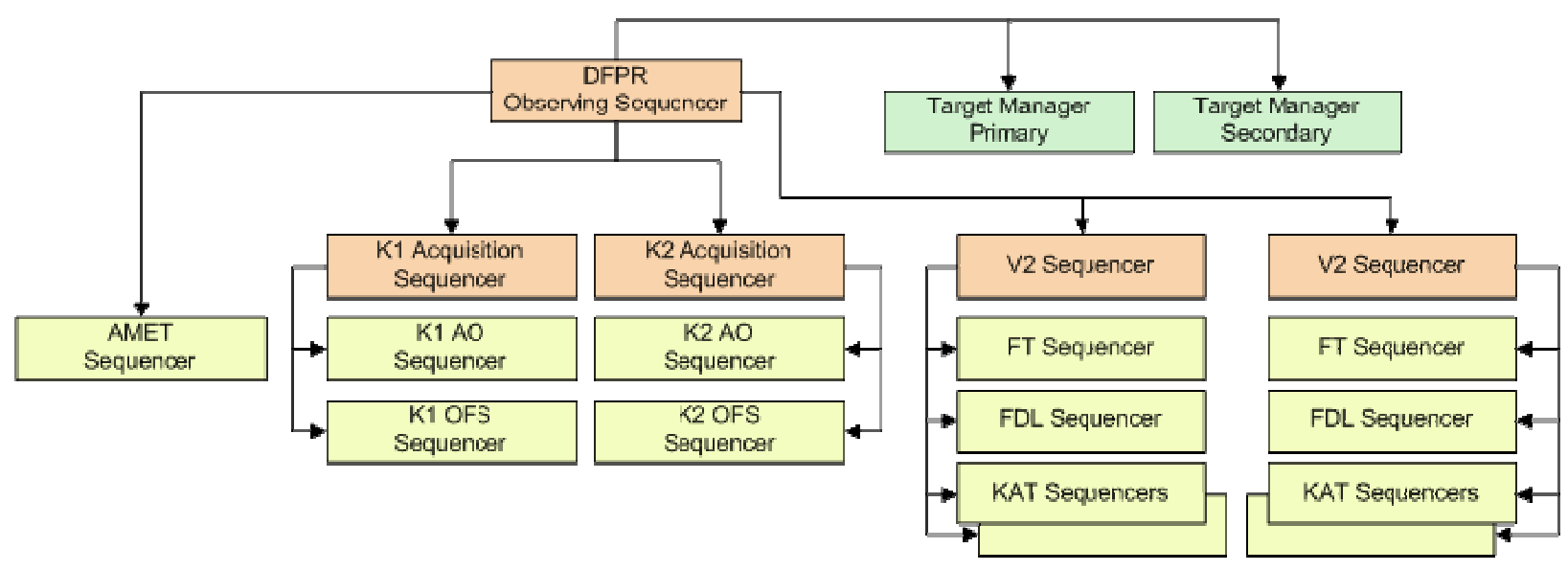

Figure 2: A layout of the observing sequencers

L-band sequencer GUI underwent the following changes:

- Functions were modified to minimize telescope moves and to optimize the sequence

- The GUI implementation was updated for improved clarity and to reduce night-time operational errors 


\subsection{Upgrades to the Real-Time Software Infrastructure: Tuning for Slow Clocks/Feedforward}

In the $\mathrm{V}^{2}$-DFPR mode, the $\mathrm{KI}$ is tracking two different targets on the two fringe trackers. The fringe tracker on the brighter object is considered a fast fringe tracker, stabilizing the optical path difference for the other fringe tracker, a slow spectrograph. These two targets are "swapped" between the beam trains by the sequencer to provide astrometric measurements.

To perform the swapping between which fringe tracker was on the brighter target and which was on the fainter target, the fringe tracker gizmo required upgrades. Additionally, considerable tuning was done to make the tracking on both fringe trackers smooth.

- During testing, the fringe tracker receiving the hold command from the other fringe tracker had its error position jump when the hold transitioned. This required a change to how the "release hold" was sent to the receiving fringe tracker, to send a start time. This way the receiving fringe tracker waits a full servo cycle (ZABCD read) before releasing its hold after commanded. This change was implemented without affecting the interface used by the Nuller gizmo, which "holds" the fringe trackers from the Nuller sequencer - an instantaneous hold/release.

- For the very slow fringe tracker clocks (slower than a configurable value - currently the system uses $10 \mathrm{~Hz}$ ), the FDL dither implementation was modified to use a modulation, rather than the PZT motor.

- To disable parametric oscillators (PO) when the clock loaded brings the servo rate too low for PO to be effective, we modified the fringe tracker parametric oscillators.

\subsection{Auto Alignment Improvements}

Beam train alignment from the telescope (AO enclosure) to the science cameras in the basement ( 150m path) is one of the critical elements of day time preparation for interferometer observations. Most of the complicated and time consuming alignments are automated, reducing manpower and human error, and during the past two years we continued to improve these auto-alignment (AA) scripts and alignment procedures.

- Two different switchyard dichroics can be used on the sky: the original $\mathrm{H}+\mathrm{K}$ dichroics (which route $\mathrm{H}-$ and $\mathrm{K}$ band light to the fringe tracker, and J-band light to the angle tracker) and K-only dichroics (which route K-band light to the fringe tracker, and $\mathrm{H}$ - and J-band light to the angle tracker). However, the K-only dichroics produce additional ghost images in the visible, which can mislead the automatic alignment, and thus Auto-Alignment setup scripts were modified to check which dichroics are configured and replace K-only with $\mathrm{H}+\mathrm{K}$ when necessary.

- The Auto-Alignment script was updated to allow the operator more flexibility to choose the subsequent alignment steps or to repeat the previous ones if not satisfactory.

- The secondary beam alignment script was modified for $\mathrm{V}^{2}$-DFPR mode to reset the OFS mirror image offsets before alignment. These offsets build up during angle tracking and may affect alignment if it is not set to zero.

- Most recently, the auto-alignment scripts were upgraded with new mode selections: DSM configuration and science mode. Within the DSM configurations are Nuller and ASTRA modes. In the Nuller DSM configuration only one star is acquired and the pupil is divided by a half mirror (Nuller mode) into two beams (primary and secondary) which then follow separate paths to the IF combining area. For single-beam modes, the half mirror is replaced with a full size mirror so that all the light travels via the primary coude train to the primary fringe tracker $\left(\mathrm{V}^{2}-K\right.$ mode), to the L-band camera when L-band dichroics are in beam $\left(\mathrm{V}^{2}-L\right.$-band mode), or with the $\mathrm{K} / \mathrm{N}$ beam splitters installed to both primary and secondary fringe trackers $\left(\mathrm{V}^{2}\right.$-SPR mode). In the ASTRA DSM configuration, two stars can be acquired simultaneously ( $\mathrm{V}^{2}$-DFPR mode), or only one star for $\mathrm{V}^{2}-K, \mathrm{~V}^{2}$ SPR or $V^{2}-L$-band modes. Depending on the DSM configuration, the AA scale factors are different even for the same science mode. This requires more complicated configuration and setup, so auto-alignment scripts were modified to incorporate all new science modes and DSM configurations.

\subsection{Procedural Improvements}

Alignment procedures and run preparations were continually improved during the past two years. 
- The $\mathrm{BC} 3$ bench has been reoriented by 90 degrees and is facing the same direction as both $\mathrm{BC} 1$ and $\mathrm{BC} 2$ (i.e., into the switchyard). This move, preceded by the installation of a horizontal periscope and the horizontal rotation of the K-only dichroics, reduces relative beam shear (translation). In addition the $\mathrm{BC} 3 \mathrm{~K}$-only dichroics were clocked in their mounts to match the $\mathrm{BC} 2 \mathrm{H}+\mathrm{K}$ 's wedge direction so that their angles also point in the same direction. These changes produce a more stable alignment as seen by KAT when switching between optics.

- The same ghost issue with the K-only dichroics in-beam was fixed during nighttime LDL moves. The LDL move script was modified to put the $\mathrm{H}+\mathrm{K}$ dichroics in-beam before the LDL move. References before and alignment recovery after the LDL moves are done with the $\mathrm{H}+\mathrm{K}$ dichroics. After the LDL move the script puts the correct dichroics in-beam.

- The LDL move script was modified to incorporate all science modes depending on which beam would be affected by the LDL position change.

- The interferometer checklist is continuously updated based on daytime and nighttime experience.

- Implemented a partial automation of the FATCAT clock file update as required after camera service or other actions which cause the illuminated pixels to change on the array using the known relative pixel differences among different filter/prism combinations. Python scripts were created to update all the clock files simultaneously after one basic clock file is updated and verified.

- More recently we successfully used the near-infrared metrology system (AMET), developed for the $\mathrm{V}^{2}$-DFPR and Astrometry modes of the ASTRA project, for the standard $\mathrm{V}^{2}$ and $\mathrm{V}^{2}$-SPR in place of the visible coude metrology (CT). Efforts are underway to use the system for the $\mathrm{V}^{2}-L$-band mode, too. The performance of AMET is better than CT, since the AMET, an on-axis metrology system, covers a significant part of the beamtrain in comparison to the $\mathrm{CT}$ which is an off-axial metrology system.

The configuration control process at the KI is working well in protecting the operational system, while at the same time enabling development of new operational capabilities and upgrades. The configuration control board (CCB) reviews the engineering change requests (ECR) and field change notices (FCN). There were 177 new ECR/FCNs, 105 closed and 2 cancelled since 1 July 2010 (Software: New: 151, Closed: 90, Cancelled: 2; Electronics: New: 16, Closed: 11; Cancelled: none; Mechanical: New: 10, Closed: 4, Cancelled: None; IF operations: New: None, Closed: None, Cancelled: None). All modified IF subsystems are captured and frozen in-situ then rebuilt from scratch on a separate host after every run.

\subsection{Equipment Obsolescence and Spares}

One of the increasing concerns in maintaining the Keck Interferometer is an issue typical to observatory environments worldwide. Equipment obsolescence becomes a serious problem as facilities age. Typical observatory facility lifetimes are measured in decades, much longer than commercial laboratories. Most of the Keck interferometer subsystems date from well over a decade ago, having been assembled in earlier JPL test bed versions and then transferred to Keck. Examining the inventory of interferometer equipment reveals a number of equipment suppliers that no longer exist or product lines long abandoned by their manufacturers. The pace of such obsolescence has accelerated in the last decade with faster business cycles and the increased rate of mergers in manufacturing, forcing suppliers to refresh and consolidate product lines.

The motion control industry presents a particularly severe example of this. The many motor controllers and servo motor systems used throughout the interferometer represent an increasing risk in obsolescence. The industry has nearly abandoned the brushed DC servo motor technology that was standard when the interferometer was assembled, replacing this technology with brushless motors that require new motor controller technology.

While there are pleasant exceptions, with some vendors still selling and servicing the same models found in the interferometer, in general the interferometer staff has had to become more adept with board level repair; forced to fix equipment when no spares are available. Fortunately the spares inventory of the interferometer remains quite good. The availability of a spare or two allows quick response to an equipment breakdown, with substantial time available after the run to replace or repair the failed spare without instrument downtime. 


\section{SCIENCE OPERATIONS}

The visibility $\left(\mathrm{V}^{2}\right)$ mode combines the adaptive optics corrected beams from the two Keck telescopes for measuring the fringe contrast in the near-infrared atmospheric windows. We had 17 observing runs since 1 July 2010, including 27.5 science nights $\left(22.5 \mathrm{~V}^{2}\right.$ Science \& 5 Nuller Science), 11.5 nights for interferometer engineering, and 1.25 nights for single telescope engineering. Eleven refereed articles were published from this instrument since the 2010 SPIE meeting. The highlights of the science results from the KI during this two-year period are presented in this section.

\subsection{Science Overview}

The upgraded KI capabilities addresse a wide range of astrophysical topics such as formation of stars and planets, exozodiacal dust, and the radial structure of active galactic nuclei. The science papers using KI data since SPIE 2010 are:

- First Keck Nulling Observations of a Young Stellar Object: Probing the Circumstellar Environment of the Herbig Ae star MWC 325, S. Ragland, K. Ohnaka, L. Hillenbrand, S. T. Ridgway, M. M. Colavita, R. L. Akeson, W. Cotton, W. C. Danchi, M. Hrynevych, R. Millan-Gabet, W. A. Traub, 2012, ApJ, 746, 126

- Mapping the radial structure of AGN tori, M. Kishimoto, S. F. Hoenig, R. Antonucci, F. Millour, K. R. W. Tristram, G. Weigelt, 2011, A\&A, 536, 78

- Resolving the Sub-AU-scale Gas and Dust Distribution in FU Orionis Sources, J. A. Eisner, L. A. Hillenbrand, 2011, ApJ, 738, 9

- Exozodiacal Dust Levels for Nearby Main Sequence Stars, R. Millan-Gabet, E. Serabyn, B. Mennesson, W. A. Traub, R. K. Barry, W. C. Danchi, M. Kuchner, S. Ragland, M. Hrynevych, J. Woillez, K. Stapelfeldt, G. Bryden, M. M. Colavita, A. J. Booth, 2011, ApJ, 734, 67

- The Innermost Dusty Structure in Active Galactic Nuclei as Probed by the Keck Interferometer, M. Kishimoto, S. F. Hoenig, R. Antonucci, R. Barvainis, T. Kotani, K. R. W. Tristram, G. Weigelt, K. Levin, 2011, A\&A, 527, 121

- Radial Structure in the TW Hya Circumstallar Disk, R. L. Akeson, R, Millan-Gabet, D. R. Ciardi, A. F. Boden, A. I. Sargent, J. D. Monnier, H. McAlister, T. ten Brummelaar, J. Sturmann, L. Sturmann, and N. Turner, 2011, ApJ, 728, 9

- The Structure of the $\beta$ Leonis Debris Disk, Stock, N. D., Su, K,Y. L., Liu, W., Hinz, P.M., Rieke, G.H., Marengo, M., Stapelfeldt, K.R., Hines, D.C., Trilling, D.E., 2010, ApJ, 724, 1238

- Spectro-interferometry of the Be Star $\delta$ Sco: Near-Infrared Continuum and Gas Emission Region Sizes in 2007, Millan-Gabet, R., Monnier, J. D., Touhami, Y., Gies, D., Hesselbach, E., Pedretti, E., Thureau, N., Zhao, M., ten Brummelaar, T., CHARA Group, 2010, ApJ, 723, 544

- Probing local density inhomogeneities in the circumstellar disk of a Be star using the new spectro-astrometry mode at the Keck interferometer, J.-U. Pott et al, 2010, ApJ, 721, 802

- Milli-arcsecond images of the Herbig Ae star HD 163296, S. Renard, et. al., 2010, A\&A, 519, 26

- Spatially and Spectrally Resolved Hydrogen Gas within 0.1 AU of T Tauri and Herbig Ae/Be Stars, J.Eisner, et. al., 2010, ApJ, 718, 774

The following paragraphs provide highlights from some of these papers.

\subsection{Young Stellar Object (YSO) Disks}

The spatial distribution of gas and dust in FU Orionis objects ${ }^{13}$ as well as in the accretion disks around T Tauri stars ${ }^{14}$ were studied using the spectro-interferometry technique enabled by the $\mathrm{V}^{2}$-SPR mode which combines milliarcsec spatial resolution with high spectral resolution $(\mathrm{R}=2000)$. This enables the separation of the continuum emission (arising primarily in the dust component) from gas probed by lines such as that caused by the Hydrogen $\mathrm{Br} \gamma$ transition. Also, using multi-wavelength interferometry (K, L and N bands, covering $2-12 \mu \mathrm{m}$ ), the Herbig Ae star MWC325 was studied, with the observations finding complexity in the disk structure and composition, and a diversity of disk properties among members of this class of young stars ${ }^{15}$. The KI was also used (together with data from IOTA, CHARA and VLTI) to accomplish pioneering efforts toward image reconstruction of these relatively complex circumstellar structures ${ }^{16}$. For a more evolved "transition" disk candidate, multi-wavelength spatially resolved observations $(2 \mu \mathrm{m}, 10 \mu \mathrm{m}$ and $\mathrm{mm})$ were 
used to infer a three-component model for the disk, with not only an inner gap in the optically thick disk as previously suggested, but also a gap between the inner and outer optically thick disks ${ }^{17}$.

\subsection{Exozodiacal Dust Around Nearby Solar-type Stars}

Older stars are surrounded by dust too, not primordial as in the case of young stars, but arising much later in asteroid collisions and comet evaporation. The emission scattered or thermally emitted by this dust is both interesting astrophysically (e.g., since it contains the dynamical imprint of planet-disk interactions) and a source of noise for direct exoplanet imaging techniques. The main science driver for the development of the KI Nuller instrument was to study the level of such "exozodi" dust emission that is prevalent among typical nearby stars, in order to inform the design of future space missions to directly detect and characterize exo-Earths. Over a year-long observing campaign (from February 2008 to January 2009), 32 nights were dedicated to this project, and a total of 43 stars were observed by three different teams. For one of those studies, based on a sample of 25 stars, a 3-sigma upper limit of 150 zodis was inferred for the class of 23 stars not previously known to have exozodi dust ${ }^{18}$. In addition, the debris disk around the star beta Leo was studied in detail using KIN and MMT nulling data as well as Spitzer imaging and photometry ${ }^{19}$.

\subsection{B2 Stars}

Classical Be stars are B spectral class stars with hydrogen emission lines in their spectra which are believed to arise from a circumstellar disk comprised of material ejected from the star. The SPR mode at the KI was used to study the Be star $48 \mathrm{Lib}^{20}$. The observations used the high spectral resolution of this mode to resolve several Pfund emission lines, in addition to $\mathrm{Br} \gamma$. The data suggest that the continuum and Pf-emission originates in compact regions inside the $\mathrm{Br} \gamma$ emission zone. The differential line properties and linear constraints are consistent with theoretical models of a rotationally flattened star inducing density perturbations in the disk, and lend direct support to the existence of radiusdependent disk density perturbations. The data also show decreasing gas rotation velocities at increasing stellocentric radii as expected for Keplerian disk rotation.

The Be star delta Sco was studied by combining KI and CHARA observations ${ }^{21}$. These authors found the K-band continuum emission was only marginally spatially resolved, while line emission from $\mathrm{He}$ I $\lambda 2.0583 \mu \mathrm{m}$ and $\mathrm{Br} \gamma$ $\lambda 2.1657 \mu \mathrm{m}$ was clearly detected with $\sim 10 \%$ lower visibilities than those of the continuum. The measured continuum/line flux ratio corresponds to much larger sizes for the line emission regions and will help constrain dynamical models being actively developed in order to explain the disk formation process in the $\delta$ Sco system.

\subsection{Radial Structure of Active Galactic Nuclei (AGN)}

Over the last several years, multiple groups have used the sensitivity of KI to expand the observed sample of active galactic nuclei (AGN). Near-infrared interferometry observations can directly probe the size scales of the accretion disk surrounding the black hole at the center of these objects. Three type-1 AGN's and a QSO (quasi stellar object) were observed with $\mathrm{KI}^{22}$. All objects had a high measured visibility $\left(\mathrm{V}^{2} \sim 0.8-0.9\right)$. These authors find $\mathrm{K}$-band sizes roughly equal to or slightly larger than the reverberation radius as a function of AGN luminosity, suggesting that the KI observations are partially resolving the dust sublimation region. Recent observations of an additional 4 objects ${ }^{23,24}$ expand the total sample to 8 objects, and for most objects the KI observations resolve the dust sublimation region. In this sample, which covers over $\sim 2.5$ orders of magnitudes in the UV/optical luminosity L of the central engine, the radial distribution systematically changes with luminosity.

A second team analyzed the multiple epochs of observations available for NGC $4151^{25}$ and found the measured visibilities from the three different nights are not correlated with the variations of the nuclear luminosity. These authors propose two models for the emission: 1) the visibility is dominated by emission from size scales smaller than $30 \mathrm{Mpc}$, which falls short of any dust reverberation measurement in NGC 4151 and of theoretical models of circum-nuclear dust distributions, or 2) the visibility is dominated by hot dust (>1300 K) at linear scales of about $50 \mathrm{Mpc}$. They favor the second hypothesis and also show that the KI data do not support a model where the dust emission size scale follows the nuclear variability of NGC 4151 as $R_{\text {dust }} \propto L^{0.5}$. 


\section{KI CLOSURE}

The NASA key science goal is accomplished and NASA Keck Interferometer (KI) operations funding is ending. The Keck Interferometer (KI) is scheduled to be closed at the end of semester 2012A. The current plan is to maintain the KI infrastructure for a year to make it possible to restart the operation if funding becomes available in the near future. We have one more observing run in late July (July 27-29) before the closure. We are planning on making some valuable science observations using the $\mathrm{V}^{2}$-DFPR mode during this run. We are also hopeful of using the laser guide star adaptive optics (LGS-AO ${ }^{26,27}$ ) for this final observing run to observe targets that are too faint in the visible wavelength for the natural guide star adaptive optics (NGS-AO).

\section{ACKNOWLEDGMENTS}

The W. M. Keck Observatory is operated as a scientific partnership among the California Institute of Technology, the University of California, and the National Aeronautics and Space Administration. The Observatory was made possible by the generous financial support of the W. M. Keck Foundation.

The data presented herein were obtained at the W. M. Keck Observatory, which is operated as a scientific partnership among the California Institute of Technology, the University of California, and the National Aeronautics and Space Administration. The Observatory was made possible by the generous financial support of the W. M. Keck Foundation.

The authors wish to recognize and acknowledge the very significant cultural role and reverence that the summit of Mauna Kea has always had within the indigenous Hawaiian community. We are most fortunate to have the opportunity to conduct observations from this mountain.

This material is based upon work performed for the Jet Propulsion Laboratory, California Institute of Technology, sponsored by the U.S. Government under Prime Contract NAS7-03001 between the California Institute of Technology and NASA.

\section{REFERENCES}

[1] Colavita, M. M., Wizinowich, P. L., \& Akeson, R. L., "Keck Interferometer status and plans,” Proc. SPIE, 5491, 454 (2004).

[2] Wizinowich, P., Akeson, R., Colavita, M., Gathright, J., Appleby, E., et al., "Recent progress at the Keck Interferometer," Proc. SPIE, 6268, 62680N (2006).

[3] Colavita, M. M., Serabyn, G., Wizinowich, P. L., \& Akeson, R. L., "Nulling at the Keck Interferometer," Proc. SPIE, 6268, 626803 (2006).

[4] Ragland, S., Wizinowich, P., Akeson, R., Colavita, M., Appleby, E. et al., "Recent progress at the Keck Interferometer: operations and $\mathrm{V}^{2}$ science," Proc. SPIE, 7013, 70130B (2008).

[5] Colavita, M. M., Serabyn, E., Booth, A. J., Crawford, S. L., Garcia-Gathright, J. I. et al., "Keck Interferometer nuller update," Proc. SPIE, 7013, 70130A (2008).

[6] Ragland, S., Akeson, R., Colavita, M., Millan-Gabet, R., Woillez, J. et al., "Recent progress at the Keck Interferometer," Proc. SPIE, 7734, 1 (2010).

[7] Colavita, M. M., Serabyn, E., Ragland, S., Millan-Gabet, R., Akeson, R. L., "Keck Interferometer nuller instrument performance," Proc. SPIE, 7734, 7734-28 (2010).

[8] Woillez, J., Akeson, R., Colavita, M., Eisner, J., Ghez, A. et al., "ASTRA: the astrometric and phase-referencing astronomy upgrade for the Keck Interferometer," Proc. SPIE, 7734, 7734-37 (2010).

[9] Ragland, S., Akeson, R. L., Armandroff, T., Colavita, M. M., Danchi, W. C. et al., "First L-Band Interferometric Observations of a Young Stellar Object: Probing the Circumstellar Environment of MWC 419," ApJ, 703, 22 (2009).

[10] Colavita, M. M., Serabyn, E., Millan-Gabet, R., Koresko, C. D., Akeson, R. L. et al., "Keck Interferometer nuller data reduction and On-sky performance," PASP, 121, 1120 (2009). 
[11] Woillez, J., Akeson, R., Colavita, M., Eisner, J., Millan-Gabet, R. et al., "Self-Phase-Referenced SpectroInterferometry on the Keck Interferometer," PASP, 124, 51 (2012).

[12] Woillez, J. Akeson, R., Colavita, M., Eisner, J., Millan-Gabet, R. et al., "First faint dual field phase referenced observations on the Keck Interferometer," Proc. SPIE, 8445, 13 (2012).

[13] Eisner, J. A., Hillenbrand, L. A., "Resolving the Sub-AU-scale Gas and Dust Distribution in FU Orionis Sources," ApJ, 738, 9 (2011).

[14] Eisner, J. A., Monnier, J. D., Woillez, J., Akeson, R. L., Millan-Gabet, R., Graham, J. R., Hillenbrand, L. A., Pott, J.-U., Ragland, S., Wizinowich, P., "Spatially and Spectrally Resolved Hydrogen Gas within 0.1 AU of T Tauri and Herbig Ae/Be Stars,” ApJ, 718, 774 (2010).

[15] Ragland, S.., Ohnaka, K., Hillenbrand, L., Ridgway, S.T., Colavita, M.M. et al., "First Keck Nulling Observations of a Young Stellar Object: Probing the Circumstellar Environment of the Herbig Ae Star MWC 325,” ApJ, 746, 126 (2012).

[16] Renard, S., Malbet, F., Benisty, M., Thiébaut, E., Berger, J.-P., "Milli-arcsecond images of the Herbig Ae star HD 163296," A\&A, 519, 26 (2010).

[17] Akeson, R. L., Millan-Gabet, R., Ciardi, D.R., Boden, A.F., Sargent, A.I., Monnier, J.D., McAlister, H., ten Brummelaar, T., Sturmann, J., Sturmann, L., and Turner, N, "Radial Structure in the TW Hya Circumstallar Disk," ApJ, 728, 9 (2011).

[18] Millan-Gabet, R., Serabyn, E., Mennesson, B., Traub, W.A., Barry, R.K. et al., "Exozodiacal Dust Levels for Nearby Main Sequence Stars,” ApJ, 734, 67 (2011).

[19] Stock, N.D., Su, K,Y. L., Liu, W., Hinz, P.M., Rieke, G.H. et al., "The Structure of the $\beta$ Leonis Debris Disk," ApJ, 724, $1238(2010)$.

[20] Pott, J.-U., Woillez, J., Ragland, S., Wizinowich, P. L., Eisner, J. A. et al, "Probing local density inhomogeneities in the circumstellar disk of a Be star using the new spectro-astrometry mode at the Keck interferometer," ApJ, 721, 802 (2010).

[21] Millan-Gabet, R., Monnier, J. D., Touhami, Y., Gies, D., Hesselbach, E. et al., "Spectro-interferometry of the Be Star $\delta$ Sco: Near-Infrared Continuum and Gas Emission Region Sizes in 2007," ApJ, 723, 544 (2010).

[22] Kishimoto, M. et al, "Exploring the inner region of Type 1 AGNs with the Keck interferometer," A\&A, 507, 3, L57 (2009).

[23] Kishimoto, M., Hoenig, S.F., Antonucci, R., Barvainis, R., Kotani, T., Tristram, K.R.W., Weigelt, G., Levin, K., "Mapping the radial structure of AGN tori," A\&A, 527, 121 (2011).

[24] Kishimoto, M., Hoenig, S.F., Antonucci, R., Millour, F., Tristram, K.R.W., Weigelt, G., "Mapping the radial structure of AGN tori," A\&A, 536, 78 (2011).

[25] Pott J.-U. et al, "Luminosity-variation Independent Location of the Circum-nuclear, Hot Dust in NGC 4151," ApJ, $715,2,736(2010)$.

[26] Wizinowich, P., Le Mignant, D., Bouchez, A., Campbell, R., Chin, J. et al., "The W. M. Keck LGS AO System: Overview," PASP 118, 297-309 (2006).

[27] Wizinowich, P., "Progress in laser guide star adaptive optics and lessons learned," Proc. SPIE, 8447, 13 (2012). 